(C) 1997 IEEE. Personal use of this material is permitted. Permission from IEEE must be obtained for all other uses, in any current or future media, including reprinting/republishing this material for advertising or promotional purposes, creating new collective works, for resale or redistribution to servers or lists, or reuse of any copyrighted component of this work in other works. 


\section{Efficient Computation of Amplitude and Phase Maps in Nuclear Medicine Equilibrium-Gated Cardiac Studies}

\author{
Juan José Vaquero, ${ }^{*}$ Andrés Santos, Salvador Pérez, \\ and Francisco del Pozo
}

\begin{abstract}
The Goertzel algorithm is proposed as a method to obtain the first harmonic coefficient of time activity curves from equilibrium gated cardiac studies. The coefficients are used to produce functional images. The algorithm achieves an important reduction in the number of operations and memory accesses needed to compute the coefficients.
\end{abstract}

Index Terms - Cardiovascular system, discrete Fourier transforms, discrete time filters, functional analysis, nuclear cardiography, nuclear imaging.

\section{INTRODUCTION}

Equilibrium-gated blood pool imaging of the heart is used to visualize and quantify cardiac function [1]. Quantificatio of global ventricular function is usually based on the determination of a timeactivity curve (TAC) over the left ventricle, from which parameters such as ejection fraction, stroke volume, and fillin rate can be obtained [2]. The complexity of the cardiac cycle can be presented in a more comprehensive way with functional images: images where each pixel represents the parameter of interest obtained after Fourier analysis of every individual TAC [3]. The main parameters are the amplitude and phase of the firs harmonic, from which three functional images are generated: the amplitude and phase maps, and the phase histogram.

1) The amplitude image shows the change of activity in each pixel, without regard to the timimg of these variations within the cardiac cycle. It is useful to show abnormalities of the wall movement [4].

2) The phase image represents approximately the relative variations in the timing of the movement of the heart regions. Delays of the blood circulation in different parts of the heart can be easily seen. This image may be used to detect delays in contraction and shows areas working asynchronously [5].

3) The phase histogram has two peaks in a healthy heart: the higher one arises from the activity of the ventricles; the smaller one from the atria. Between the two peaks exists a difference of approximately $180^{\circ}$. The width of each peak shows the synchronization of the contractions.

The computation of the firs harmonic coefficien of every pixel's TAC requires a number of operations, proportional to the number of time samples. The number of images per cardiac cycle is a compromise between temporal resolution and acquisition time: as the signal-to-noise ratio (SNR) depends on the Poisson statistics of

Manuscript received December 19, 1995; revised September 5, 1996. Asterisk indicates corresponding author.

*J. J. Vaquero is with the Grupo de Bioingeniería y Telemedicina, ETSI Telecomunicación, Universidad Politécnica de Madrid, Madrid 28040 Spain (e-mail: jjvl@teb.upm.es).

A. Santos, S. Pérez, and F. del Pozo are with the Grupo de Bioingeniería y Telemedicina, ETSI Telecomunicación, Universidad Politécnica de Madrid, Madrid 28040 Spain. the nuclear data acquisition for a given time, increasing the number of images per cardiac cycle means reducing the number of counts per image and the SNR accordingly. A typical study has between 16 and $32(64 \times 64$ pixels $)$ images per cycle; this represents a good compromise between spatial resolution and acquisition time. Then, 4096 TAC's have to be analyzed.

In this Communication we present an efficien method to estimate the firs TAC Fourier coefficient Several methods are available to compute the fast Fourier transform (FFT) when a limited number of coefficient are needed [6], but they are efficien only for a large number of time points. Here, however, we need to compute many FFT's with a few time points at the input; then, the reduction in the number of operations provided by fast algorithms is not significant and simpler algorithms can be more efficient as will be shown.

\section{METHOD}

Using a commercial gamma camera, images are acquired synchronously with the $\mathrm{R}$ wave and stored in the LIST mode (in this mode, acquisition coordinate pairs $x$ and $y$ for each scintillation are stored together with a time reference in a list format [7], [8]). After cine-sequence reconstruction, a TAC is obtained for every pixel. To minimize the quantization noise, and due to the finit number of points obtained (one from each image) in typical studies, temporal interpolation or smoothing is needed for good representation of the TAC's. From the different interpolation methods available, cubic spline interpolation is chosen due to its global smoothness, that produces a function continuous up to its second derivative. The smoothing step is needed only to display the TAC's prior to computation of the functional images, since the coefficient themselves generate the smoothest version of the TAC's.

The amplitude and phase of the firs harmonic is the firs Fourier coefficien of each raw TAC, and can be obtained by different methods: directly from the discrete Fourier transform (DFT) formula

$$
X(k)=\sum_{n=0}^{N-1} x(n) W_{N}^{k n}
$$

where $W_{N}=e^{-j 2 \pi / N}$, or with any algorithm that computes the FFT. We propose to use the Goertzel algorithm because it has several advantages, as will be shown.

Once the coefficient have been calculated, the amplitude and phase maps can be produced. The generation of the amplitude map is straightforward, but for the phase maps two observations can be made.

1) Points with negligible amplitude should not be represented in the phase map as these points are usually static (extracardiac) structures. A mask that includes the points with amplitude less than $10 \%$ of the maximum value in the image can be used to skip such points.

2) The phase image should preserve the periodic nature of the phase values, thus, a cyclic color scale has to be used. There should be also the possibility to shift this scale when the boundary between two colors is near a peak in the phase histogram: a more meaningful representation can be obtained if similar values are represented in the same color. 


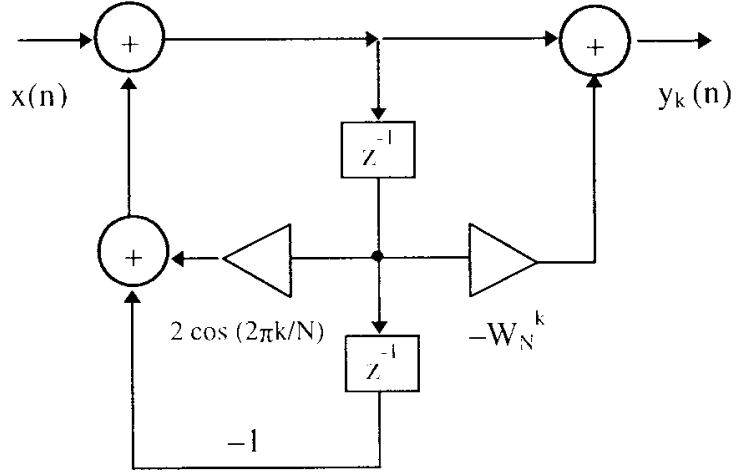

Fig. 1. Implementation of the Goertzel algorithm as a linear filter

\section{A. The Goertzel Algorithm}

The Goertzel algorithm is an efficien way to compute a reduced number of DFT values [9]. It can be implemented as a linear filterin operation with only two complex coefficients To derive it, (1) can be expressed as

$$
X(k)=\sum_{n=0}^{N-1} x(n) W_{N}^{-k(N-n)}
$$

where the second term has been multiplied by $W_{N}^{-k N}$ (which is always equal to one). Equation (2) can also be seen as the $N$ th value of convolution of the $N$ samples of $x(n)$ with a filte that has the impulse response

$$
h_{k}(n)=W_{N}^{-k n} u(n) .
$$

Then $X(k)$ is simply the output of this filte at time $N$, when its input is $x(n)$

$$
X(k)=\left.x(n) \otimes h_{k}(n)\right|_{n=N} .
$$

Thus, to compute the DFT parameters, a filte could be implemented with the system function expressed as

$$
H_{k}(z)=\frac{1}{1-W_{N}^{-k} z^{-1}}=\frac{1-W_{N}^{k} z^{-1}}{1-2 \cos (2 \pi k / N) z^{-1}+z^{-2}} .
$$

The direct form implementation of the filte is shown in Fig. 1. As can be seen, only one real coefficien and one complex coefficien are needed. As just the $N$ th value of the output is needed, the forward branch of the filte has to be computed only at time $N$.

\section{RESUlts}

Tables I-III show the number of real operations (noncomplex multiplications or additions) needed to compute the DFT coefficient from a noncomplex input signal. The split-radix algorithm [10] is the best among nine different ways to compute the DFT [11]. Except for the FFT algorithm, the tables reflec the number of operations performed on the computation of a single (complex) coefficien (as needed in this application to produce the amplitude and phase maps). The split-radix algorithm produces the $N$ FFT coefficients and thus in this case the figure given are the number of operations needed to compute the $N$ coefficients Table I shows the number of nontrivial real multiplications (multiplications by $1,-1, j$, and $-j$ are excluded). Table II shows the number of real additions.

Table III shows one of the main advantages of the Goertzel algorithm: it needs just one real and one complex coefficien while the computation of the DFT needs $N$ complex coefficient (this table excludes again the trivial coefficients)
TABLE I

Number of Nontrivial Real Multiplications For Different Algorithms (N/A: Not Applicable)

\begin{tabular}{cccc}
\hline$N$ & $\begin{array}{c}\text { Split-radix } \\
\text { FFT }\end{array}$ & $\begin{array}{c}\text { DFT } \\
\text { (per coef.) }\end{array}$ & $\begin{array}{c}\text { Goertzel } \\
\text { (per coef.) }\end{array}$ \\
\hline 16 & 10 & 24 & 14 \\
24 & N/A & 40 & 22 \\
32 & 34 & 56 & 30 \\
\hline
\end{tabular}

TABLE II

Number of ReAl AdDitions for Different Algorithms

\begin{tabular}{cccc}
\hline$N$ & $\begin{array}{c}\text { Split-radix } \\
\text { FFT }\end{array}$ & $\begin{array}{c}\text { DFT } \\
\text { (per coef.) }\end{array}$ & $\begin{array}{c}\text { Goertzel } \\
\text { (per coef.) }\end{array}$ \\
\hline \multirow{2}{*}{16} & 60 & 30 & 32 \\
24 & N/A & 46 & 48 \\
32 & 164 & 62 & 64 \\
\hline
\end{tabular}

TABLE III

Number of Real Coefficients Needed for Each Algorithm

\begin{tabular}{ccc}
\hline$N$ & DFT & $\begin{array}{c}\text { Goertzel } \\
\text { (per coef.) }\end{array}$ \\
\hline 16 & 24 & 3 \\
24 & 40 & 3 \\
32 & 56 & 3 \\
\hline
\end{tabular}

\section{Discussion AND CONCLUSIONS}

From the previous tables, it can be seen that the Goertzel algorithm is an efficien way to compute the amplitude and phase maps. Time savings are $34 \%$ with a 486 central processing unit (CPU), and go up to $40 \%$ with a Pentium. When these savings are applied to the computation of the individual time-activity curves (4096 for $64 \times 64$ pixels images; 16384 for $128 \times 128$ images), the reduction in the number of operations becomes significant For example, for a sequence with 24 images $(64 \times 64$ pixels $)$, the direct DFT method needs 163840 real-number multiplications while with the Goertzel algorithm this figur is reduced to 90112 . This reduction of CPU cycles together with the reduction of memory access allows implementation of functional image analysis on smaller computers with reasonable execution times. The Goertzel algorithm has the additional advantage of being efficien for any $N$ (time points), while FFT-based methods lose efficienc when $N$ is not an integral power of two.

In a practical clinical case, we have measured that our algorithm takes $3 \mathrm{~s}$ for a 32 -image sequence, $128 \times 128$ pixels per image, on a Sun $4 / 370$ system, while the same process in our standard equipment used in clinical routine needs between 15 and $30 \mathrm{~s}$, depending on the CPU used. In both cases, the coefficient obtained are exactly the same.

The proposed method will be more useful with functional maps obtained from magnetic resonance images. In that case, the number of individual TAC's will be in the order of $256 \times 256$, and hence the reduction in the number of operations will be very significant

\section{REFERENCES}

[1] C. Schiepers and J. J. Almasi, "Equilibrium gated blood pool imaging at rest and during exercise," in Effective Use of Computers in Nuclear Medicine, M. J. Gelfand, S. R. Thomas, Eds. New York: McGraw-Hill, 1988.

[2] S. Bacharach and M. Green, "Data processing in nuclear cardiology: measurement of ventricular function," IEEE Trans. Nucl. Sci., vol. 29, no. 4, pp. 1343-1354, Aug. 1982. 
[3] S. Bacharach, M. Green, D. Vitale, G. White, M. Douglas, R. Bonow, and S. Larson, "Optimum Fourier filterin of cardiac data: A minimumerror method," J. Nucl. Med., vol. 24, pp. 1176-1184, 1983.

[4] J. Candell and D. Ortega, Cardiologia Nuclear. Barcelona, Spain: Ediciones Doyma, 1992.

[5] M. Green and S. Bacharach, "Functional imaging of the heart: Methods, limitations and examples from gated blood pool scintograph," Progress Cardiovasc. Diseases, vol. 28., no. 5, pp. 319-348, Mar/Apr. 1986.

[6] H. Sorensen and S. Burrus, "Efficien computation of the DFT with only a subset of input or output points," IEEE Trans. Signal Processing, vol. 41, no. 3, pp. 1184-1200, Mar. 1993.

[7] S. Bacharach, M. Green, and J. Borer, "Instrumentation and data processing in cardiovascular nuclear medicine: Evaluation of ventricular function," Seminars in Nucl. Med., vol. 9, no. 4, pp. 257-274, Oct. 1979.

[8] J. Lear and J. Pratt, "Real-time list-mode processing of gated cardiac blood pool examinations with forward-backward framing", Eur. J. Nucl. Med., vol. 19, pp. 177-180, 1992.

[9] J. Proakis and D. Manolakis, Digital Signal Processing Principles, Algorithms and Applications. New York: Macmillan, 1992.

[10] P. Duhamel, "Implementation of 'Split-Radix' FFT algorithms for complex, real, and real-symmetric data," IEEE Trans. Acoust., Speech, Signal Processing, vol. ASSP-34, no. 2, pp. 285-295, Apr. 1986.

[11] H. Sorensen, D. Jones, M. Heideman, and S. Burrus, "Real-valued fast Fourier transform algorithms," IEEE Trans. Acoust., Speech, Signal Processing, vol. ASSP-35, no. 6, pp. 849-863, June 1987. 